\title{
OBTENÇÃO DE NANOCOMPÓSITO MAGNÉTICO PARA REMOÇÃO DE METAIS PESADOS
}

\section{Theodoro da Rosa Salles'; Franciele da Silva Bruckmann², Ivana Zanella da Silva $^{3}$, Sergio Robeto Mortari ${ }^{3}$, Cristiano Rodrigo Bohn Rhoden ${ }^{4}$}

\section{RESUMO}

O crescimento da atividade industrial trouxe inúmeras tecnologias para o mercado nacional, gerando empregos e oportunidades. Contudo, esta é uma das principais responsáveis pela geração de resíduos de metais pesados que ao serem direcionados a corpos d'água ajudam no crescimento de doenças. Junto a isso, os métodos de tratamento de efluentes empregados atualmente, muitas vezes, não apresentam uma significativa eficiência na remoção destes microcontaminantes, o que faz com que seja necessário o desenvolvimento de novas técnicas de tratamento. O objetivo deste trabalho é obter um nanocompósito magnético, contendo partículas de óxido de grafeno e quitosana, para remoção de metais pesados. O nanocompósito magnético foi obtido utilizando uma metodologia simples, eficiente e de baixo custo. A remoção dos metais pesados foi avaliada utilizando ICP-OES e ICP-MS. Mediante as caracterizações foi possível confirmar a obtenção do nanocompósito magnético (GO·CS$\left.\cdot \mathrm{Fe}_{3} \mathrm{O}_{4}\right)$, bem como sua eficiência na remoção dos metais.

Palavras-chave: Óxido de grafeno; Quitosana; Ferrita; Microcontaminantes.

Eixo Temático: Tecnologia, Inovação e Desenvolvimento Sustentável (TIDS).

\section{INTRODUÇÃO}

A atividade industrial é uma das principais responsáveis pelas inovações presentes no mercado. Segundo o CNI (Confederação Nacional da Indústria), ela

\footnotetext{
${ }^{1}$ Autor/Apresentador - Acadêmico do curso de Engenharia Química -Universidade Franciscana-UFN. E-mail: theodoro.rsalles@gmail.com

${ }^{2}$ Mestranda do Programa de Pós-graduação em Nanociências- Universidade Franciscana - UFN. Email: francielebruckmann2@gmail.com

${ }_{3}^{3}$ Professores do Programa de Pós-graduação em Nanociências- Universidade Franciscana - UFN. Emails: ivanazanella@gmail.com e mortari@ufn.edu.br.

${ }^{4}$ Orientador - Professor do Programa de Pós-graduação em Nanociências- Universidade Franciscana - UFN. E-mail: cristianorbr@gmail.com
} 
representa $20 \%$ do PIB. Contudo, esta é uma das maiores geradoras de resíduos de metais pesados, que são potencialmente agressores aos seres humanos e ao meio ambiente. Estes microcontaminantes, quando direcionados a corpos d'água, e em excesso, contribuem para disseminação de doenças e problemas fisiológicos (WANG; LUAN; WU, 2019). Desta forma, muitas indústrias buscam formas eficientes e de baixo custo para remoção destes metais de seus efluentes.

Os métodos de tratamento de efluentes empregados atualmente, tais como coagulação e floculação, muitas vezes não apresentam resultados significativos, e em alguns casos acabam gerando resíduos que necessitam serem tratados elevando o custo operacional nas estações de tratamentos (MOREIRA et al., 2019). Assim, consequentemente eles podem atingir o meio ambiente e comprometer o ecossistema, cultivo de plantas e culturas agrícolas, e retornarem ao consumo humano (BELTRAME; LHAMBY; BELTRAME, 2016).

Em decorrência disso, diversas pesquisas estão sendo desenvolvidas na busca de minimizar os problemas causados pelos microcontaminantes, considerando e ecotoxicológica (CAMPOS, 2018). Dentre as alternativas, a adsorção demonstra-se uma excelente opção na remoção de metais pesados presentes na água.

A adsorção é uma operação unitária, na qual ocorre a deposição do adsorbato (contaminante) sobre a superfície do adsorvente (GEANKOPLIS; HERSEL; LEPEK, 2018). O sucesso deste processo depende das características do adsorvente, como estrutura química, área superficial e morfologia (TONUCCI, 2014). Junto a isso, a nanotecnologia surge com um conjunto de técnicas para manipulação da matéria em escala atômica e molecular (FAHNING; LOBÃO, 2011). Um fator que tem sido amplamente estudado em estudos de adsorção é a utilização de adsorventes magnéticos, uma vez que estes possibilitam a reutilização do material e diminuição de custos operacionais, sendo possível evitar as etapas de filtração e centrifugação do processo (RHODEN et al., 2021).

O óxido de grafeno $(\mathrm{GO})$ (figura 1) é um material 2D derivado do grafeno que possui elevada área superficial. Obtido a partir da esfoliação e oxidação química do grafite, ele apresenta grupos funcionais oxigenados em sua estrutura, como 
carboxilas, hidroxilas, carbonilas e epóxi (RHODEN et al., 2021). Por apresentar características importantes para a adsorção, O GO tem sido bastante utilizado na remoção de metais pesados e formação de nanocompósito.

Figura 1 - Estrutura do óxido de grafeno.

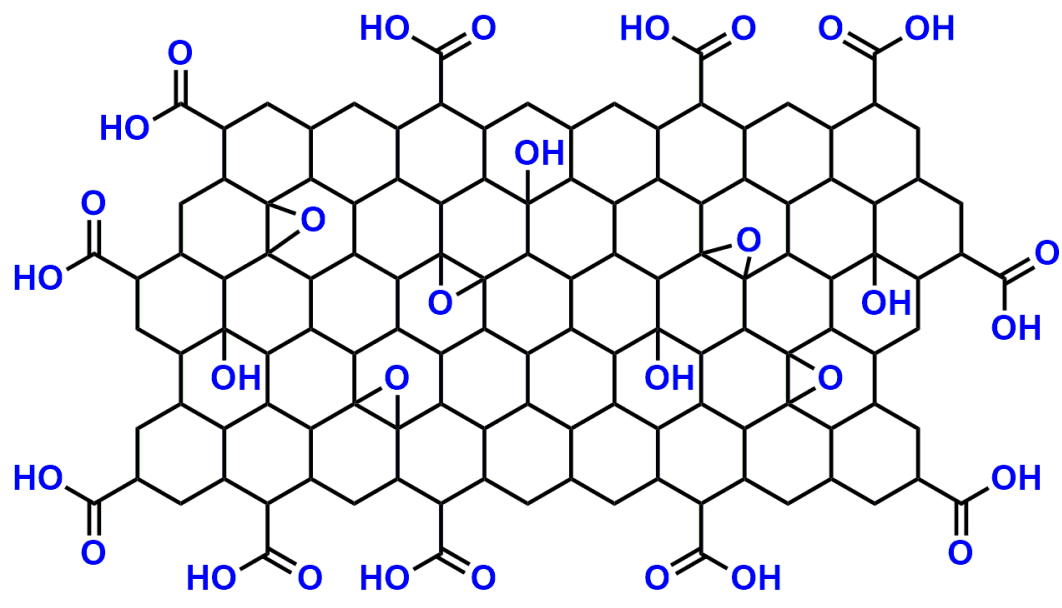

Fonte: construção do autor.

A quitosana (NCS) (figura 2) é um biopolímero obtido através da desacetilação da quitina (RASOULUZADEHZALI; NAMAZI, 2018). Trata-se de um nanomaterial catiônico que possui amina e hidroxila como grupos funcionais, que conferem a ela características com elevada sensibilidade a pH e biocompatibilidade, sendo possível utilizá-la na formação de nanocompósitos. Sendo ela um biopolímero de baixo custo e atóxico, apresenta uma ótima relação custo-benefício com ampla possibilidade de utilização como agente farmacêutico e na remoção de micropoluentes aquáticos (KUMAR et al., 2017).

Figura 2 - estrutura química da nanoquitosana.

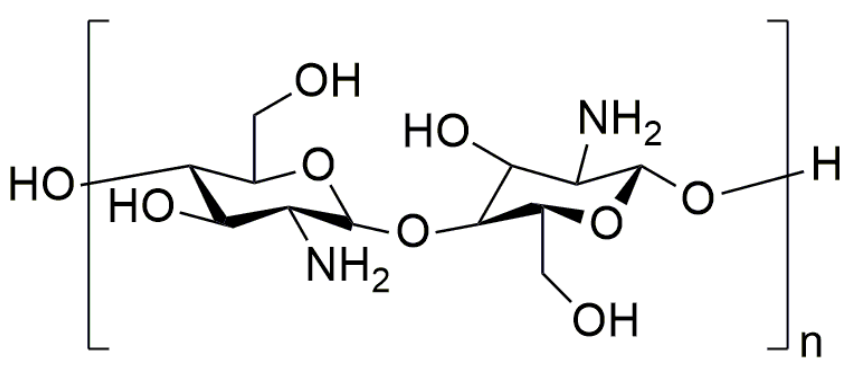

Fonte: construção do autor. 
O GO e a CS têm sido amplamente utilizados em conjunto. Recentemente, Li e seus colaboradores (2019) sintetizaram um hidrogel de GO-CS-álcool polivinílico para adsorção de $\mathrm{Cd}^{2+}$ e $\mathrm{Ni}^{2+}$, em uma síntese fácil e econômica.

O presente trabalho tem como objetivo, sintetizar um nanocompósito magnético ( $\mathrm{GO} \cdot \mathrm{CS} \cdot \mathrm{Fe}_{3} \mathrm{O}_{4}$ ) para remoção de metais pesados de uma água residuária obtida de uma indústria metalmecânica da cidade de Santa Maria/RS.

\section{MATERIAIS E MÉTODOS}

\subsection{MATERIAIS}

No desenvolvimento deste trabalho foram utilizados os reagentes comerciais e equipamentos de análise listados a seguir: Acetona - Synth® (Brasil); Ácido clorídrico 37\% - Synth® (Brasil);Ácido Sulfúrico 98\% - Synth® (Brasil); Água milli-q Millipore® (Alemanha); Grafite - Sigma® (Estados Unidos); Balança analítica AUW 220D - Shimadzu® (Japão); Balão de fundo redondo - Ilmabor® (Alemanha); Balão de fundo redondo - Poliglass $\AA^{\circledR}$ (Brasil); Béquer de vidro - Laborglas ${ }^{\circledR}$ (Brasil); Capela de exaustão - Permution® (Brasil); Espectrofotômetro de infravermelho - Spectro One, Perkin Elmer (EUA); Estufa de esterilização - J Prolab® (Brasil); Ultrassom Unique® (Brasil); pHmetro - Denver Instrument $\AA^{\circledR}$ (China); Rotaevaporador - Fisaton $\AA$ (Brasil); Difratometro de Raios-X - D2 Phase, Bruker® (Alemanha).

\subsection{OBTENÇÃO DO NANOCOMPÓSITO MAGNÉTICO}

Para a magnetização do nanocompósito, adiciona-se a um balão de $1000 \mathrm{~mL}$, contendo $500 \mathrm{~mL}$ de água Milli-Q® previamente desoxigenada, a CS e GO previamente preparados, cloreto de ferro II $\left(\mathrm{FeCl}_{2}\right)$ e hidróxido de amônio $\left(\mathrm{NH}_{4} \mathrm{OH}\right)$ para ajustar o pH $(\mathrm{pH}=9)$. A mistura é então submetida a irradiação ultrassônica por 120 minutos (RHODEN, et al; 2017). Sequencialmente, aproxima-se o balão a um campo magnético externo de maneira que o produto obtido fique retido no fundo do balão, podendo ser lavada consecutivamente com metanol e acetona, sem perda de material. Por fim, o material é levado a estufa a $80^{\circ} \mathrm{C}$ durante 20 minutos para a evaporação dos solventes remanescentes (BRUCKMANN, et al., 2020).

\subsection{REMOÇÃO DOS METAIS PESADOS}


A metodologia utilizada neste estudo na coleta preliminar da água residuária de uma indústria metalmecânica da cidade de Santa Maria -RS. As amostras foram coletas em pequenas quantidades utilizando frascos de polietileno com capacidade de $1 \mathrm{~L}$ e mantidos sofre refrigeração, conforme as regras estabelecidas pela ABNT NBR 9.897 e 9.898.

Para o procedimento de adsorção foram realizados ensaios em laboratório para avaliar a eficiência do nanocompósito sintetizado. As alíquotas coletadas, nos tempos 0 e 180 minutos, foram analisadas conforme metodologia descrita abaixo.

Os elementos químicos Cobalto (Co), Ferro (Fe), Manganês (Mn), Nickel (Ni), Vanádio (V) e Zinco (Zn), foram determinados pela Espectrometria de emissão óptica com plasma indutivamente acoplado (ICP-OES) utilizando o equipamento Spectro Ciros CDD, SPECTO Analytical Instruments (Alemanha). As linhas espectrais selecionadas foram Co $(228,616 \mathrm{~nm})$, Fe $(238,204 \mathrm{~nm})$, Mn $(257,611 \mathrm{~nm})$, $\mathrm{Ni}(231,604 \mathrm{~nm}), \mathrm{V}(292,464 \mathrm{~nm})$ e Zn $(213,856 \mathrm{~nm})$. Os elementos químicos Cádmio (Cd), Cromo (Cr) e Cobre (Cu) foram determinados por Espectroscopia de massa com Plasma Indutivamente Acoplado (ICP-MS) utilizando o equipamento Elan DRC II, PerkinEImer Sciex (Canada). As razões $\mathrm{m} / \mathrm{z}$ de íons monitorados foram Cd (112), Cr (52) e Cu (63).

\subsection{CARACTERIZAÇÕES}

A difração de raios X (DRX), mede diretamente a distância e o ângulo entre os planos paralelos do reticulo cristalino. As análises de difração de raios- $X$ foram realizadas utilizando o difratômetro da marca Bruker, modelo D2 Phaser, localizado na Central Analítica da Universidade Franciscana - UFN. As amostras foram maceradas e dispostas no porta-amostras de forma que ficassem com a superfície mais lisa possível (RHODEN, 2018).

Outra técnica instrumental bastante utilizada é a espectrocopia de infravermelho fornece evidências da presença de grupos funcionais em estruturas orgânicas. O equipamento utilizado para caracterizar os nanomateriais sinteziados até o momento foi FTIR da Perkin-Elmer, modelo Spectro One. As pastilhas foram obtidas com $2 \mathrm{mg}$ de amostra e $200 \mathrm{mg}$ do suporte (KBr). O espectro aparece, em 
forma de bandas, resultante das vibrações das moléculas ao absorverem a radiação infravermelha (RHODEN, 2018).

\section{RESULTADOS E DISCUSSÕES}

\subsection{NANOCOMPÓSITO GO·NCS· $\mathrm{Fe}_{3} \mathrm{O}_{4}$}

A figura 3 apresenta os difratogramas de raios-X da nanoquitosana, óxido de grafeno e do nanocompósito $\mathrm{GO} \cdot \mathrm{CS} \cdot \mathrm{Fe}_{3} \mathrm{O}_{4}$.

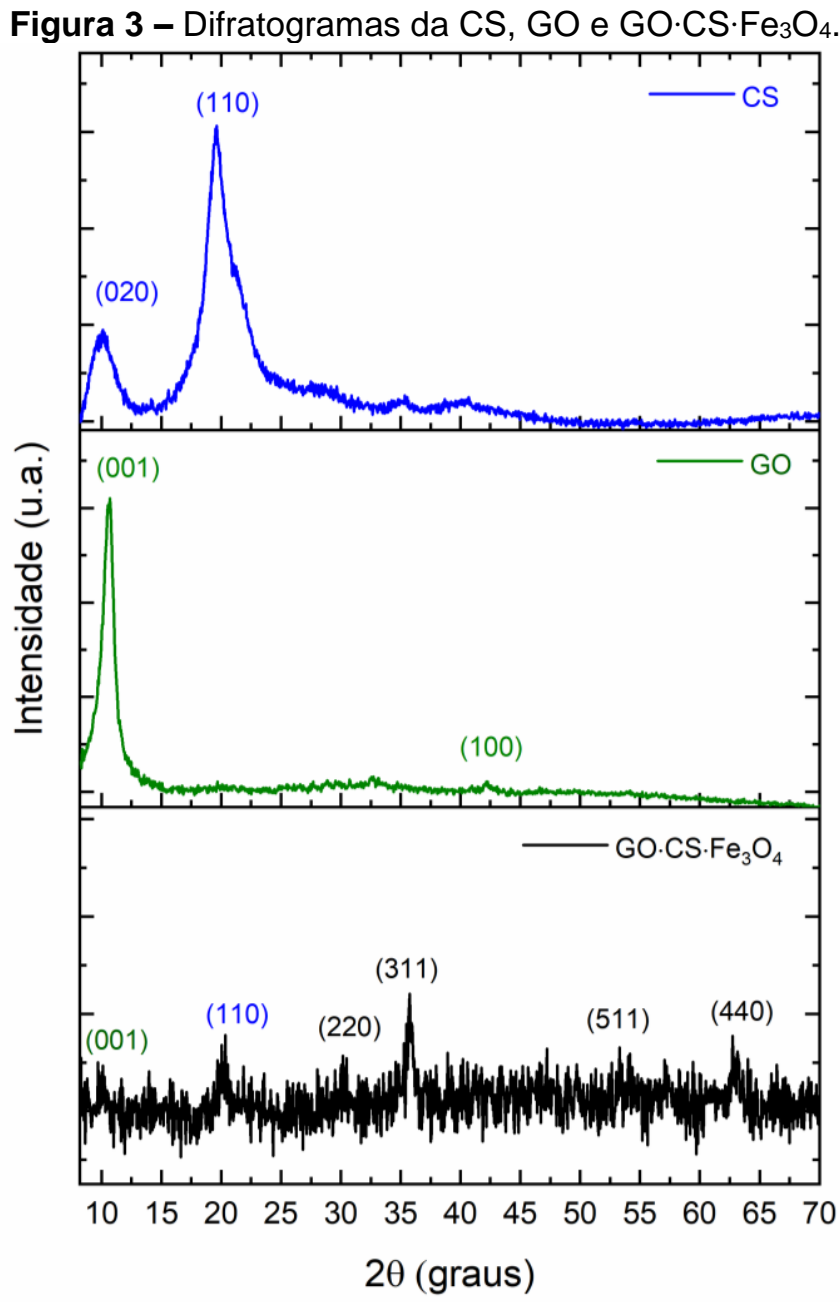

Fonte: Construção do autor.

A partir dos resultados de DRX obtidos para a quitosana, é possível observar dois picos $2 \theta \approx 10,5^{\circ}$ e $22,50^{\circ}$, característicos dos planos (020) e (110) respectivamente, corroborando com os resultados encontrador por Dey et al (2017). O difratograma do GO mostra uma completa oxidação e esfoliação do grafite, 


\section{Q PUFN}

ausência do pico em $2 \theta \approx 26^{\circ}$ e presença do pico em torno de $2 \theta \approx 11,7^{\circ}(001)$, característico da formação do óxido de grafeno. O surgimento do pico em $2 \theta \approx 41^{\circ}$ (100) revela uma pequena distância entre as camadas do nanomaterial (SALLES et al., 2020).

$\mathrm{O}$ resultado de difração de raios-X do $\mathrm{GO} \cdot \mathrm{CS} \cdot \mathrm{Fe}_{3} \mathrm{O}_{4}$ apresenta um picos em torno de $2 \theta \approx 11,7^{\circ}$ e $20,50^{\circ}$, indicando a presença de $\mathrm{GO}$ e CS respectivamente. Os picos em $2 \theta \approx 30^{\circ}(220), 35^{\circ}(311), 57,2^{\circ}$ (511) e $63^{\circ}(440)$ denotam a presença de nanoparticulas de $\mathrm{Fe}_{3} \mathrm{O}_{4}$ (SHAHBAZI; MARNANI; SALAHSHOOR, 2019).

A figura 4 aresenta os espectros de infravermelho (FTIR) da quitosana, óxio de grafeno e do nanocompósito.

Figura 4 - Espectroscopia de infravermelho de CS, GO e GO.CS. $\mathrm{Fe}_{3} \mathrm{O}_{4}$

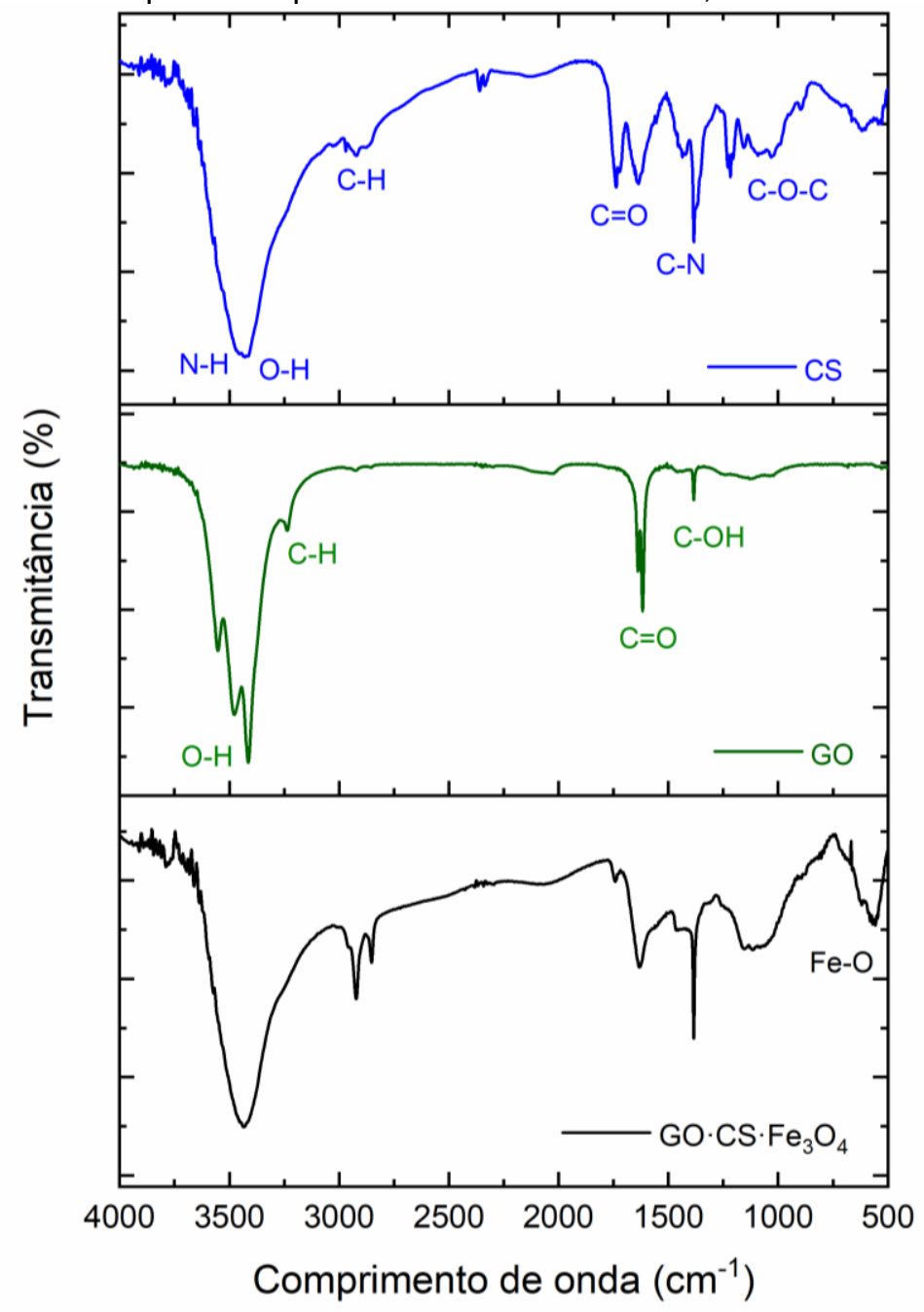

Fonte: Construção do autor. 
O FTIR da quitosana apresenta uma banda intensa em $3432 \mathrm{~cm}^{-1}$, característica das vibrações de alongamento dos grupos $\mathrm{N}-\mathrm{H}$ e $\mathrm{O}-\mathrm{H}$, em $2925 \mathrm{~cm}^{-1}$ indica o estiramento da ligação $\mathrm{C}-\mathrm{H}, 1630 \mathrm{~cm}^{-1}$ representa a ligação entre $\mathrm{C}=\mathrm{O}$, $1400 \mathrm{~cm}^{-1}$ denota a presença das ligações C-N e $1031 \mathrm{~cm}^{-1} \mathrm{o}$ estiramento C-O-C (ZULFIKAR et al, 2016). Já para o GO, a banda mais intensa em $3407 \mathrm{~cm}^{-1}$ representa o estiramento $\mathrm{O}-\mathrm{H}$, indicando a presença de grupos funcionais $\mathrm{OH}$ e $\mathrm{COOH}$ na estrutura do nanomaterial. A banda em $3227 \mathrm{~cm}^{-1}$ motra o alongamento da ligação $\mathrm{C}-\mathrm{H}, 1636 \mathrm{~cm}^{-1}$ o alongamento vibracional de $\mathrm{C}=\mathrm{O}$ e em $1387 \mathrm{~cm}^{-1}$ denota a presença de grupos C-OH (SALLES et al., 2020) (RHODEN et al., 2021).

O FTIR do nanocompósito GO.CS $\cdot \mathrm{Fe}_{3} \mathrm{O}_{4}$, além das bandas mencionadas anteriormente dos nanometeriais CS e GO, há a presença da banda em $588 \mathrm{~cm}^{-1}$, características de partículas de $\mathrm{Fe}_{3} \mathrm{O}_{4}$, indicando sua presença no nanocompósito obtido (RHODEN et al., 2021).

\subsection{REMOÇÃO DOS METAIS PESADOS}

A tabela 1 mostra os resultados obtidos após aplicação do tratamento utilizando o nanocompósito magnético, bem como o padrão CONAMA 430/2011.

Tabela 1 - Remoção dos contaminantes metálicos em água residuária (amostra real).

\begin{tabular}{cccc}
\hline Parâmetro & $\begin{array}{c}\text { Amostra } \\
\text { Bruta }\end{array}$ & Amostra Tratada & Padrão CONAMA 430/2011 \\
\hline Cádmio total $\left(\mathrm{mg} \cdot \mathrm{L}^{-1}\right)$ & 0,00748 & $<\mathrm{LQ}$ & 0,2 \\
Cromo total $\left(\mathrm{mg} \cdot \mathrm{L}^{-1}\right)$ & 0,578 & 0,0043 & 1,10 \\
Cobre dissolvido $\left(\mathrm{mg} \cdot \mathrm{L}^{-1}\right)$ & 0,126 & 0,0205 & 1,00 \\
Cobalto total $\left(\mathrm{mg} \cdot \mathrm{L}^{-1}\right)^{*}$ & 0,025 & 0,0013 & 0,05 \\
Ferro dissolvido $\left(\mathrm{mg} \cdot \mathrm{L}^{-1}\right)$ & 4,82 & 0,410 & 15,00 \\
Manganês dissolvido $\left(\mathrm{mg} \cdot \mathrm{L}^{-1}\right)$ & 1,01 & 0,1270 & 1,00 \\
Níquel total $\left(\mathrm{mg} \cdot \mathrm{L}^{-1}\right)$ & 0,81 & 0,0610 & 2,00 \\
Vanádio total $\left(\mathrm{mg} \cdot \mathrm{L}^{-1}\right)^{*}$ & 13,2 & 0,0038 & 0,10 \\
Zinco total $\left(\mathrm{mg} \cdot \mathrm{L}^{-1}\right)$ & 5,29 & 0,0419 & 5,00 \\
\hline
\end{tabular}

Fonte: Construção do autor.

Uma vez que a concentração dos contaminantes na amostra tratada é mais baixa que os limites encontrados na regulamentação em questão (CONAMA 
430/2011), é possível verificar a eficiência do nanocompósito na remoção destes micropoluentes aquáticos. A alta remoção destes metais pesados da amostra bruta, indica uma excelente capacidade de adsorção do nanomaterial obtido neste estudo.

\section{CONCLUSÃO}

A partir dos resultados obtidos ao decorrer deste trabalho, é possível confirmar a obtenção do nanocompósito $\mathrm{GO} \cdot \mathrm{CS} \cdot \mathrm{Fe}_{3} \mathrm{O}_{4}$, bem como sua eficiência na remoção dos metais pesados. A partir destes resultados iniciais, serão realizados novos testes de adsorção, bem como estudos da variação do pH da solução, concentração do adsorvente, estudo cinético e termodinâmico, afim de encontrar a melhor condição para que o processo ocorra.

\section{AGRADECIMENTOS}

Os autores agradecem a FAPERGS, a CAPES e a Universidade Franciscana UFN pelas bolsas concedidas.

\section{REFERÊNCIAS}

BELTRAME, T. F.; LHAMBY, A. R; BELTRAME, A.; Efluentes, resíduos sólidos e educação ambiental: Uma discussão sobre o tema. Revista Eletrônica em Gestão, Educação e Tecnologia Ambiental, v. 20, n. 1, p. 351-362, 2016.

BRUCKMANN, F. S. et al. Synthesis, characterization and cytotoxicity evaluation of magnetic nanosilica in L929 cell line. Disciplinarum Scientia| Naturais e Tecnológicas, v. 21, n. 3, p. 1-14, 2020.

CAMPOS, A. C. V. Otimização e validação do método para análise de microcontaminantes de preocupação emergente por cromatografia gasosa acoplada à espectrometria de massas utilizando derivatização online. Dissertação de Mestrado (PPGQUIM), Universidade Federal de Ouro Preto, 2018

DEY, S. C. et al. Preparation, characterization and performance evaluation of chitosan as an adsorbent for remazol red. International Journal of Latest Research in Engineering and Technology, v. 2, n. 2, p. 52-62, 2016.

FAHNING, B. M.; LOBÃO, E. B. Nanotecnologia aplicada a fármacos. 2011. Disponível em: < http://www.ucv.edu.br/fotos/files/06.pdf> Acesso em: 10 de Agosto de 2018. 
GEANKOPLIS, C. J.; HERSEL, A. A.; LEPEK, D. H. Transport Processes and Separation Process Principles. 5 th . Ed. New York: Pearson Education, 2018.

KUMAR, R. S. et al. Nanochitosan modified glass ionomer cement with enhanced mechanical properties and fluoride release. International journal of biological macromolecules, v. 104, p. 1860-1865, 2017.

$\mathrm{LI}, \mathrm{C}$. et al. Adsorption of $\mathrm{Cd} 2+$ and $\mathrm{Ni2}+$ from aqueous single-metal solutions on graphene oxide-chitosan-poly (vinyl alcohol) hydrogels. Langmuir, v. 35, n. 13, p. 4481-4490, 2019.

MOREIRA, A. C. O.; SALLES, T. R.; SILVA, I. Z.; RHODEN, C. R. B. Desenvolvimento de um protocolo de remoção de metais pesados acoplado a uma estação de tratamento. XXIII Simpósio de Ensino, Pesquisa e Extensão - SEPE, 2019, Santa Maria/RS.

RASOULUZADEHZALI, M.; NAMAZI, $H$. Facile preparation of antibacterial chitosan/graphene oxide-Ag bio-nanocomposite hydrogel beads for controlled release of doxorubicin. International Journal of Biological Macromolecules, v. 116, p. 54-63, 2018.

RHODEN, C. R. B. et al. Study from the influence of magnetite onto removal of hydrochlorothiazide from aqueous solutions applying magnetic graphene oxide. Journal of Water Process Engineering, v. 43, p. 102262, 2021.

RHODEN, C. R. B. et al. Síntese fácil e direta do óxido de grafeno magnético. Disciplinarum Scientia, v. 18, n. 2, p. 389-397, 2017.

RHODEN, C. R. B. Síntese de análogos de peptídeos incorporados a materiais nanoparticulados de carbono funcionalizados via reações multicomponentes. 2018. 107f. Tese de Doutorado (Doutor em Nanociências), Universidade Franciscana, 2018.

SALLES, T. R. et al. Graphene oxide optimization synthesis for application on laboratory of Universidade Franciscana. Disciplinarum Scientia Naturais e Tecnológicas, v. 21, n. 3, p. 15-26, 2020.

SHAHBAZI, A.; MARNANI, N. N.; SALAHSHOOR, Z.. Synergistic and antagonistic effects in simultaneous adsorption of $\mathrm{Pb}$ (II) and $\mathrm{Cd}$ (II) from aqueous solutions onto chitosan functionalized EDTA-silane/mGO. Biocatalysis and Agricultural Biotechnology, v. 22, p. 101398, 2019.

TONUCCI, M. C. Adsorção De Diclofenaco, Estradiol E Sulfametoxazol Em Carvões Ativados E Nanotubos De Carbono: Estudos Cinéticos E Termodinâmicos. p. 109f. Dissertação de Mestrado (Programa de Pós-Graduação em Engenharia Ambiental), Univiersidade Federal de Ouro Preto, 2014. 
WANG, C.; LUAN, J.; WU, C. Metal-organic frameworks for aquatic arsenic removal. Water research, v. 158, p. 370-382, 2019.

ZULFIKAR, M. A. et al. Preparation of Fe3O4-chitosan hybrid nano-particles used for humic acid adsorption. Environmental Nanotechnology, Monitoring \& Management, v. 6, p. 64-75, 2016. 